\title{
475622 - OBSERVATIONAL STUDY OF A NEWLY DEVELOPED SUPRAGLOTTIC AIRWAY
}

\author{
Dietrich Henzler, MD PhD ${ }^{2}$, Rolf Rossaint, Professor, MD PhD ${ }^{1}$, Gereon Schaelte, \\ MD MBA ${ }^{1}$
}

\section{Anesthesiology, University Hospital of the RWTH Aachen, Aachen, Germany 2. Anesthesia and Critical Care, Dalhousie University, Halifax, NS, Canada}

Introduction: Supraglottic airway devices (SAD) are increasingly used in routine anesthesia. The i-gel (Intersurgical) is a single-use SAD without inflatable cuff. The soft, elastomer-gel made cuff mirrors the perilaryngeal anatomical structures. Theoretically, it is kept in place by adhesion forces as opposed to the conventional LMA, which seals by the pressure exerted to the perilaryngeal tissue.

Methods: After approval from the institutional REB, a cohort of patients scheduled for elective surgery in general anaesthesia with a SAD, were consented. Exclusion criteria were age $<18$, BMI $>30$, history of regurge or hiatal hernia, planned operation time $>2$ hours or extreme posturing, e.g. prone position. The type of anaesthesia and the medication used was left to the discretion of the consultant anaesthesiologist. Patients were ventilated in a volume controlled mode with a tidal volume of $8 \mathrm{ml} / \mathrm{kg} \mathrm{BW}$ and a PEEP 3 cmH2O. Insertion times, reposition maneuvers and injuries were recorded, as well as ventilatory parameters and leakage. The ease of insertion was graded on a 5 stage score from very easy to impossible. Blood spurs on removal of the i-gel were noted and the patients were interviewed for postanaesthetic complications of hoarseness, soar throat or globus feeling in the recovery room and on the ward.

Results: Prelminary results from 29 patients (11 male) with a mean age of $46 \pm 20$ years, a mean BMI of $26 \pm 6$ are presented. $65 \%$ of patients were ASA I or II status, and $96,6 \%$ were graded Mallampati I or II. The mean anaesthesia time was $64 \pm 35$ minutes. Insertion was performed in $13,8 \%$ by medical students, in $24.1 \%$ by residents and in $62.1 \%$ by staff anesthesiologist. Insertion was judged very easy in $51.7 \%$, as easy in $41.4 \%$ and as complicated in 2 cases (6.9\%). Two times the SAD had to be exchanged. In one case, after 8 unsuccesful attemps to place the i-gel, endotracheal intubation was performed. In another case, after turning the patient in the lateral decubitus position, a non-correctable leakage occurred and the i-gel was exchanged for a conventional LMA, which provided sufficient seal to continue the procedure. No case of blood spurs or injuries to the oral area was noted. One patient complained of hoarseness in the recovery room, otherwise the i-gel was very well tolerated. The size of the i-gel used correlated with body weight, but not with the BMI.

Discussion: In this first evaluation the device was easy to handle and provided a good seal, as long as the patient's head was kept in orthograd position. Movements of the head increased the risk of leakage, sometimes the device just slipped out of the mouth.

Fixation with tape was effective in all but 2 cases to solve the problem. From its design, the rigid handle with the incorporated bite block suggests simple and fault-free insertion even by lay persons after short instruction. So theoretically, the i-gel could be better than conventional LMA or endotracheal intubation in pre-hospital use by non-medical or lay 
first response helpers. The non-pressure cuff suggest less tissue trauma to the perilaryngeal structures than conventional LMA, increasing patient's post anaesthetic comfort and reducing post-op disorders. Further studies should be undertaken to address these questions.

References: Sharma S, Scott S, Rogers R, Popat M. The i-gel airway for ventilation and rescue intubation. Anaesthesia. $2007 ; 62: 419-20$. 